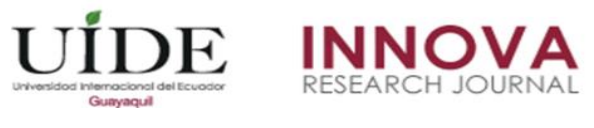

INNOVA Research Journal, ISSN 2477-9024

(Mayo, 2017). Vol. 2, No.5 pp. 137-147

DOI: https://doi.org/10.33890/innova.v2.n5.2017.237

URL: http://revistas.uide.edu.ec/index.php/innova/index

Correo: innova@uide.edu.ec

\title{
El diagnóstico organizacional, contextualizado en los negocios fabriles de la provincia de Santa Elena-Ecuador 2015-2016
}

\section{The organizational diagnosis, contextualized in the manufacturing businesses of the province of Santa Elena-Ecuador 2015-2016}

Econ. Luis Asencio Cristóbal

Ing. Steve Freddy Guarnizo Crespo

Universidad de Guayaquil, Ecuador

Econ. Willian Caiche Rosales

Universidad Estatal Península de Santa Elena, Ecuador

Ing. Viviana Vanessa Medina Vergara

Universidad Internacional del Ecuador, Ecuador

Autores para correspondencia: luis.asencioc@ug.edu.ec, steve.guarnizoc@ug.edu.ec, caichewilliam@yahoo.com, vimedinave@internacional.edu.ec

Fecha de recepción: 28 de Febrero de 2017 - Fecha de aceptación: 28 de Abril de 2017

Resumen: El objetivo de este reporte científico consiste en identificar los principales inconvenientes organizacionales que tienen los negocios fabriles, en los 3 cantones de la provincia de Santa Elena-Ecuador. La metodología a seguir para realizar un diagnóstico organizacional a profundidad se sustenta en métodos y técnicas científicas (entrevistas y encuestas), en el estudio realizado a 119 pymes fabriles de un total de 653 empresas productoras, en los ámbitos: productivo, organizacional y comercialización interna y externa. La concepción de diagnóstico está relacionada a la acción de realizar investigaciones que permitan buscar información para poder describir un fenómeno o incluso encontrar las causas de su ocurrencia. El diagnóstico tiene como propósito reflejar la situación de un cuerpo, estado o sistema para que luego se proceda a realizar una acción o tratamiento que ya se preveía realizar o que a partir de los resultados del diagnóstico se decide llevar a cabo. Los resultados obtenidos arrojan las principales insuficiencias de costobeneficio, estructura organizacional y procesos de comercialización, que permitirán elaborar e implementar en futuras investigaciones, las estrategias empresariales para contribuir a lograr un desarrollo productivo sostenible en las empresas fabriles de la provincia de Santa Elena, resaltando la necesidad del uso crítico y reflexivo de la teoría a lo largo del proceso investigativo en el campo de las Ciencias Empresariales.

Palabras clave: diagnóstico organizacional; fabril; desarrollo empresarial

Abstrat: The objective of this scientific report is to identify the major organizational problems that have manufacturing businesses, in the province of Santa Elena-Ecuador 3 cantons. The methodology to follow to make an organizational diagnosis to depth is based on methods and scientific techniques (interviews and surveys), study 119 manufacturing SMEs of a total of 653producing companies, in the areas: productive, organizational and internal and external marketing. The concept of diagnosis is related to action research that allow information to be able to describe a phenomenon or even to find the causes of their occurrence. The diagnosis is intended to reflect the situation of a body, State or system so that then proceed to perform than already anticipated to 
perform or that based on the results of the diagnosis is decided to carry out an action or treatment. The results shed major shortfalls of cost-benefit, organizational structure and processes of marketing, that will develop and implement in future research, business strategies to help achieve a sustainable productive development in the manufacturing companies of the province of Santa Elena, highlighting the need for critical and reflexive use of the theory throughout the investigative process in the field of business science. Key words: differential organizational manufacturing business development.

Key words: differential organizational; manufacturing; business development.

\section{Introducción}

Desde una perspectiva de análisis en el contexto empresarial podemos plantear que el diagnóstico adquiere dimensiones mucho más complejas porque se trata de investigar problemas empresariales como la producción, comercialización y desarrollo organizacional, en función del nivel de instrucción de los empresarios y de la coyuntura de las políticas gubernamentales del país. El empresario tiene la necesidad de conocer su entorno organizacional, para orientar adecuadamente las actividades productivas, necesarias para el desarrollo empresarial y sobre todo para que todos logren un nivel de productividad adecuado a las exigencias del mercado nacional e internacional.

Las intervenciones buscaran profundizar en el diagnóstico previo para actuar y generar un pronóstico de resultados fiable que permita actuar con seguridad; con este fin se realiza una medición de las variables y elementos que se quieren modificar. (Hernández, 2012)

Por lo tanto, después de completar un análisis SWOT (FODA) los gerentes están listos para formular las estrategias apropiadas que explotan las fortalezas y las oportunidades externas de una organización, amortiguan o protegen a la organización de amenazas externas o corrigen debilidades críticas. (Robbins, 2010)

Para entender el proceso administrativo y su aplicación en las empresas, es fundamental entender a aquel como un sistema con objetivos determinados, que funciona gracias a insumos, procesos productivos, productos (resultados), que se "autorregulan" por la evaluación continua de su funcionamiento, gracias a los controles. El enfoque de sistema es holístico (totalizador) porque las partes del proceso administrativo son elementos interactuantes; las causas y los efectos repercuten entre ellos. (Rodríguez, 2012).

Para (Romagnoli, 2014) el diagnóstico empresarial "constituye una herramienta sencilla y de gran utilidad a los fines de conocer la situación actual de una organización y los problemas que impiden su crecimiento, sobrevivencia o desarrollo. Es un proceso de varios estudios realizados en las empresas de producción, servicios y de comercio. Nos permite identificar y conocer una serie de problemas para plantear un plan de acción que oriente el porvenir de la organización." De igual modo, los autores de este reporte consideran, que se diagnostica para saber el nivel de logros alcanzados por la empresa, identificar debilidades y fortalezas, así como las causas que impiden el desarrollo y los factores que pueden acelerar el logro del objetivo, es decir qué precisa ser atendido, modificado, en función de los objetivos planteados. 
Dentro de la comprensión de los aspectos empresariales, entre ellos, el diagnóstico empresarial, desempeña una función esencial el enfoque de sistema, que proporciona la orientación general para su estudio, como una realidad totalizadora, formada por un conjunto de objetos unidos por alguna forma de interacción o interdependencia entre ellos. Por esta razón, el conocimiento del enfoque de sistema es de capital importancia para la investigación en el área del conocimiento de las Ciencias Empresariales.

Un sistema no es un conjunto de elementos reunidos mecánicamente, sino un todo organizado y complejo, esto significa, cualidades generales inherentes al conjunto, las cuales se diferencian de las características individuales de los componentes que lo integran. Es justamente la interacción entre los componentes del sistema lo que genera sus cualidades integrativas generales.

El diagnóstico organizacional, al igual que todos los aspectos empresariales, están sujetos a leyes que los caracterizan como sistema. De aquí, la importancia que presenta el estudiar las cualidades generales de los sistemas para el dominio de la metodología de la investigación empresarial. Estas cualidades son las siguientes: componentes, estructura, y relaciones funcionales del sistema.

\section{Componentes del sistema}

Considerando esta perspectiva de análisis, el estudiar el proceso administrativo por medio del método sistémico, implica tomar en consideración sus componentes fundamentales: planeación, organización, dirección y control como categorías fundamentales de dicho proceso.

\section{Estructura de sistema}

La característica de mayor estabilidad del sistema es su estructura, posibilitando que éste mantenga su integridad, a pesar de los cambios que se pueden producir entre sus componentes y su entorno. En términos empresariales, un sistema considera las entradas: recursos, materiales, información que pasan a una transformación o procesamiento, obteniendo como resultado una salida: bien o servicio, que es mejorado mediante la retroalimentación permanente.

\section{Relaciones funcionales del sistema}

Las funciones de un sistema dependen de su estructura. El diagnóstico organizacional debe revelar las funciones que cumple como parte de un sistema. Estas se deben analizar como resultantes de las actividades que realizan los elementos que lo integran, las relaciones funcionales que existen entre sus componentes, y entre estos y el contexto en su totalidad.

Las relaciones funcionales pueden ser de dos tipos: De coordinación y de subordinación. El primero se refiere a que las funciones de los componentes del sistema deben estar coordinadas entre sí y el segundo se puede observar en dos dimensiones. Por un lado se produce entre los componentes del sistema y por otra parte la subordinación de funciones es la que encontramos entre el sistema estudiado y el todo complejo a que pertenece. En este caso, el sistema estudiado 
(diagnóstico organizacional) es considerado como un subsistema que cumple determinadas funciones dentro de un sistema mayor (proceso administrativo).

En concordancia con esta corriente de pensamiento y fundamentación teórica ofrecemos los resultados cuantitativos más representativos de los métodos empíricos empleados en el diagnóstico organizacional realizado en las empresas fabriles de los Cantones Santa Elena, Libertad y Salinas de la provincia Santa Elena.

\section{Diagnostico organizacional}

Una de las aplicaciones del diagnóstico organizacional, más adoptado por las empresas es la que va encaminada al desarrollo organizacional, el cual es un proceso de cambio estrictamente planeado con base al conocimiento que se tiene acerca de la situación por la que atraviesa la organización y la evaluación de los resultados después de la implementación de las propuestas.

Es muy importante establecer el objetivo que se persigue con el diagnóstico, pues permite conocer la situación real de la organización en un momento dado para descubrir problemas y áreas de oportunidad, con el fin de corregir los primeros y aprovechar las segundas. El diagnóstico no es un fin en sí mismo, sino que es el primer paso esencial para perfeccionar el funcionamiento comunicacional de la organización y conducir de forma científica el desarrollo productivo y organizacional de las empresas. A criterio de los autores, el diagnóstico tiene un carácter de resultado y a la vez de proceso, para ello debe compararse cómo se está desarrollando lo planificado en relación con los resultados que se van obteniendo en concordancia con los objetivos trazados por la organización.

Los elementos más comunes del diagnóstico, que plantean diferentes autores, son los siguientes: Sistemático, Dinámico, Profundo e Integral. El diagnóstico debe ser sistemático (metódico) porque el objeto de diagnóstico está en constante cambio y desarrollo, por lo que no es posible analizar de manera aislada algún componente del objeto, ya que no permite tener una visión completa del mismo. Por ello se requiere de un diagnóstico integral con un enfoque de sistema. Por otro lado del análisis, el diagnóstico debe ser dinámico porque los cambios que se producen en el objeto de diagnóstico son impredecibles, sobre todo si se trata de aspectos económicos empresariales, por lo que el diagnóstico debe tener la posibilidad de adaptarse a esos tipos de cambios y asumirlos sin que varíen sustancialmente sus resultados. De igual manera el diagnóstico debe ser profundo ya que mientras más se profundice en las características en estudio, mayores posibilidades habrá de asumir medidas ya sea para potenciar esas características o para tratar de reducir sus efectos o disminuir las mismas. El enfoque integral, propicia el conocimiento real de la estructura y dinámica del funcionamiento de la empresa. Esta concepción holística implica comprender el desarrollo organizacional como un sistema de contenidos y funciones relativamente estable, que participa activamente en la regulación y autorregulación de la empresa. Estas características son las que permitieron encontrar las insuficiencias en los procesos administrativos de los 119 negocios fabriles en la provincia de Santa Elena. 
En el diagnóstico organizacional se diferencian tres etapas principales a saber: i) Generación de información; ii) Organización de la información; y iii) Perspectivas del diagnóstico organizacional.

En la generación de información, se observa a su vez tres aspectos: La forma en que se recolecta la información, las herramientas y los procesos utilizados; La metodología utilizada para recopilar la información, la cual sigue dos corrientes, los métodos usados para obtener información desde el cliente (entrevistas, cuestionarios) y los usados para obtenerla desde el consultor (observación) y la frecuencia con que se recolecta la información, la cual depende de la estabilidad del sistema. En la organización de la información, se considerar aspectos claves, como: El diseño de procedimientos para el proceso de la información; Almacenamiento apropiado de los datos y ordenamiento de la información, de modo que sea fácil de consultar y Análisis e interpretación de la información, que consiste en separar los elementos básicos de la información y examinarlos con el propósito de responder a las cuestiones planteadas al inicio de la investigación. El diagnóstico organizacional se divide en dos perspectivas principales, una funcional y otra cultural, cada una con sus propios objetivos, métodos y técnicas. Son complementarias entre sí y dan origen a dos tipos de diagnóstico: Diagnóstico funcional y diagnóstico cultural.

El diagnóstico funcional (su nombre debido a una perspectiva funcionalista) examina principalmente las estructuras formales e informales de la comunicación, las prácticas de la comunicación que tienen que ver con la producción, la satisfacción del personal, el mantenimiento de la organización, y la innovación, mientras que el diagnóstico cultural es una sucesión de acciones cuya finalidad es descubrir los valores y principios básicos de una organización, el grado en que éstos son conocidos y compartidos por sus miembros y la congruencia que guardan con el comportamiento organizacional. Se evidencia que con la aplicación de la metodología empleada, los resultados obtenidos permitieron detectar las principales insuficiencias o carencias en lo organizacional, comercial y eficiencia productiva de 119 negocios fabriles de las 653 empresas productoras de la provincia de Santa Elena.

Podemos deducir desde esta perspectiva teórica empresarial que: diagnosticar implica describir el desarrollo organizacional de las empresas, buscando sus aspectos relevantes, tanto en los factores causales como en las condiciones en que se produce. Al mismo tiempo implica pronosticar e intervenir oportunamente. Estas constituirán sus funciones básicas, sin embargo, una de las falacias de los teorías estudiadas es que no asumen como aspectos esenciales el análisis del fenómeno en toda su extensión y adolecen del análisis de las interrelaciones que tiene éste con otros fenómenos que pueden surgir como variables ajenas del proceso investigativo. Se corrobora que en el análisis realizado, se evidenciaron los aspectos fundamentales identificados en el proceso de ejecución del diagnóstico empresarial

\section{Metodología}

\section{Diseño de la investigación}

En la realización del diagnóstico organizacional en los negocios fabriles de la provincia Santa Elena se decidió emplear un enfoque cuali-cuantitativo. La investigación cualitativa "tiene 
como finalidad la descripción de las cualidades y características de un fenómeno; tan solo pretende estudiar una parte de la realidad y no buscar probar teorías o hipótesis, sino descubrir las cualidades del objeto de estudio" (Muñoz, 2011).

Por ello, se logró concebir las particularidades en los aspectos relevantes de la organización, producción y comercialización de las pymes; utilizando la técnica de la observación, a través del acercamiento directo con los fabricantes.

El enfoque cuantitativo se basa en medir "las características de los fenómenos sociales, lo cual supone derivar de un marco conceptual pertinente al problema analizando una serie de postulados que expresen relaciones entre las variables estudiadas de forma deductiva. Este método tiende a generalizar y normalizar resultados" (Bernal, 2010)

Para el efecto, se diseñaron las encuestas dirigidas a los fabricantes de bienes en los 3 cantones de la provincia de Santa Elena. Los instrumentos de recolección de datos, permitieron receptar y procesar información cuantificable a través de los cuestionarios aplicados. Con esta metodología, se realiza la revisión y análisis teórico de las tendencias actuales del objeto de estudio (dificultades empresariales referidas en el instrumento aplicado en el diagnóstico organizacional), la observación real de lo concebido teóricamente en contraste con el objeto de estudio y su comportamiento en el tiempo.

A partir de estas concepciones se realiza la exploración empírica cuali-cuantitativa de carácter no probabilístico y se obtienen los resultados del diagnóstico siguiendo la metodología que se ilustran en la figura 1.

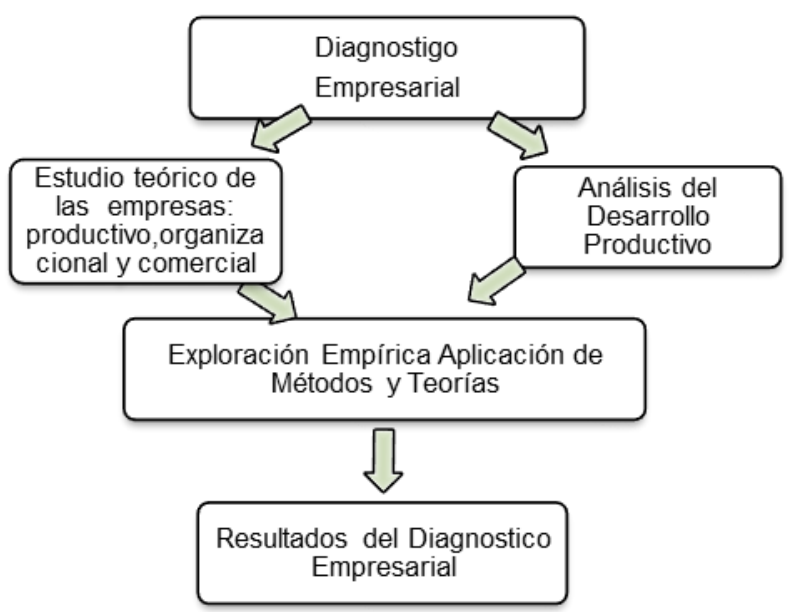

Fig. 1: Ilustración de la metodología seguida para realizar el diagnóstico organizacional en los negocios fabriles de la Provincia de Santa Elena (Elaboración propia)

\section{Población y muestra}

Para el desarrollo del trabajo, se tomó en consideración a 653 empresas fabriles de la provincia Santa Elena, que representan la población. A partir de esta población, se determina la muestra de 114 pymes cuantificada para esta investigación, obtenida por medio de la fórmula 
aplicable a estos casos de población finita. La cantidad de negocios fabriles de la provincia Santa Elena, se muestran en la siguiente tabla:

Tabla 1: Población y muestra - Negocios fabriles de la Provincia de Santa Elena por cantones.

\begin{tabular}{|c|l|r|r|r|}
\hline$N^{\mathbf{0}} .-$ & \multicolumn{1}{|c|}{ DETALLE } & $\begin{array}{c}\text { Negocios } \\
\text { fabriles en la } \\
\text { provincia }\end{array}$ & $\begin{array}{c}\text { Participación } \\
\text { porcentual }\end{array}$ & $\begin{array}{c}\text { Muestra: } \\
\text { Numero de } \\
\text { encuestas a } \\
\text { aplicarse }\end{array}$ \\
\hline 1.- & Cantón Santa Elena & 109 & $16,69 \%$ & 21 \\
\hline 2.- & Cantón La Libertad & 401 & $61,41 \%$ & 70 \\
\hline 3.- & Cantón Salinas & 143 & $21,90 \%$ & 28 \\
\hline \multicolumn{2}{|c|}{ Total de fabricantes } & 653 & $100 \%$ & 119 \\
\hline
\end{tabular}

\section{Resultados}

A continuación se muestran los resultados obtenidos sobre las dificultades empresariales más significativas en las organizaciones que fabrican bienes en la provincia de Santa Elena, derivados de la aplicación de los métodos empíricos (Encuestas y entrevistas) empleados en el proceso de investigación.

Tabla 2: Principales dificultades en los negocios fabriles de la Provincia Santa Elena. Provincia de Santa Elena

\begin{tabular}{|c|c|c|c|c|c|}
\hline \multicolumn{6}{|c|}{ Provincia de Santa Elena } \\
\hline $\begin{array}{c}\text { Principales dificultades } \\
\text { empresariales }\end{array}$ & $\begin{array}{c}\text { Totalmente } \\
\text { en desacuerdo }\end{array}$ & $\begin{array}{c}\text { En } \\
\text { desacuerdo }\end{array}$ & $\begin{array}{c}\text { Ni de acuerdo } \\
\text { ni en } \\
\text { desacuerdo }\end{array}$ & De acuerdo & $\begin{array}{l}\text { Totalmente } \\
\text { de acuerdo }\end{array}$ \\
\hline Escasa inversión & 8 & 16 & 13 & 37 & 45 \\
\hline $\begin{array}{c}\text { Dificultad para adquirir } \\
\text { maquinarias }\end{array}$ & 11 & 16 & 17 & 36 & 39 \\
\hline Pago excesivo de impuestos & 9 & 13 & 39 & 28 & 30 \\
\hline $\begin{array}{l}\text { Escaza socialización de } \\
\text { normas ambientales }\end{array}$ & 17 & 10 & 16 & 29 & 47 \\
\hline $\begin{array}{l}\text { Poca publicidad para } \\
\text { comercializar }\end{array}$ & 3 & 10 & 25 & 39 & 42 \\
\hline
\end{tabular}




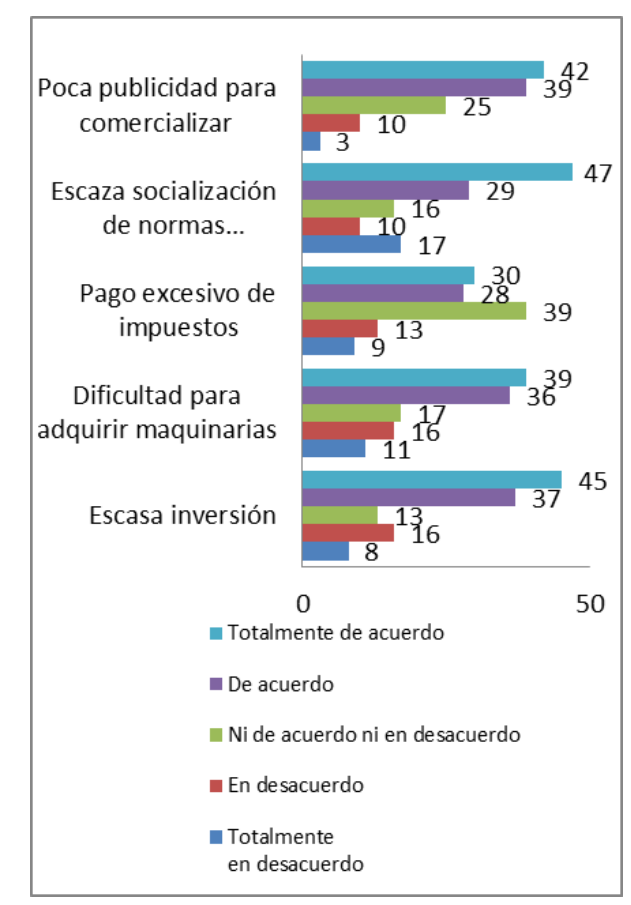

Fig. 2: Ilustración de las principales dificultades empresariales

\section{Discusión de Resultados}

Se desprende de este análisis de la muestra de 119 fabricantes, que las dificultades empresariales más significativas para ser investigadas son: 1) Escaza socialización de normas ambientales con 47 encuestados; 2) Escasa inversión con 45 encuestados; 3) Poca publicidad para comercializar con 42 encuestados; 4) Dificultad para adquirir maquinarias con 39 encuestados. Desde esta perspectiva de análisis y dentro del diagnóstico de dificultades realizado, se consideró oportuno conocer el nivel de instrucción de los fabricantes referentes a conocimientos en las diferentes áreas de la administración de empresas, para poder estar en condiciones de contribuir a la solución de las necesidades organizacionales más significativas explicadas anteriormente.

En la obtención de estos resultados se emplearon métodos empíricos, destacándose la aplicación y procesamiento de Encuestas aplicadas a 119 empresarios fabricantes de la provincia Santa Elena, para conocer el nivel de instrucción asociado con el conocimiento que tienen sobre la conducción del proceso administrativo, cuyos resultados se ilustran en la Tabla 3.

Provincia de Santa Elena

\section{Tabla 3. Nivel de instrucción e insuficiencia de conocimiento de los empresarios de la Provincia de Santa Elena}




\section{Provincia de Santa Elena}

\begin{tabular}{llllll} 
Instrucción & Santa Elena & La Libertad & Salinas & Total & \% \\
\hline Primaria & 1 & 14 & 2 & 17 & $14,29 \%$ \\
Secundaria & 12 & 43 & 18 & 73 & $61,34 \%$ \\
$\begin{array}{l}\text { Superior } \\
\text { Total }\end{array}$ & 8 & 13 & 8 & 29 & $24,37 \%$ \\
& 21 & 70 & 28 & 119 & $100 \%$ \\
Insuficiencia en conocimiento de: & Totalmente & En & Ni de acuerdo & De & $\begin{array}{l}\text { Totalmente } \\
\text { de acuerdo }\end{array}$ \\
& en & desacuerdo & ni en & acuerdo & \\
Realizar planes estratégicos & desacuerdo & & 1 & 37 & 76 \\
$\begin{array}{l}\text { Optimizar uso de maquinarias e } \\
\text { insumos }\end{array}$ & 2 & 3 & 4 & 37 & 72 \\
Capacitar a trabajadores & 0 & 4 & & 33 & 84 \\
$\begin{array}{l}\text { Fomentar los procesos } \\
\text { productivos }\end{array}$ & 0 & 0 & 2 & 29 & 80 \\
\begin{tabular}{l} 
Socializar leyes para exportación \\
\hline
\end{tabular} & 3 & 0 & 10 & 33 & 74 \\
\hline
\end{tabular}

Se infiere de los resultados obtenidos y representados en la Tabla 3, que la insuficiencia de conocimiento por parte de los empresarios atendiendo al nivel de instrucción para la conducción y administración de una empresa (principalmente en las áreas: productiva, comercialización y desarrollo organizacional), se aprecian los siguientes resultados: i) primaria con $14.29 \%$; ii) Secundaria con $61.34 \%$ y iii) Superior con $24.37 \%$, que refleja la limitada preparación para el manejo organizacional de los empresarios de nuestra provincia, ya que sólo el $24.37 \%$ tiene preparación académica universitaria, en tanto que el 61.34\% tiene instrucción de bachillerato. Esta situación es una de las principales limitantes del desarrollo productivo y organizacional.

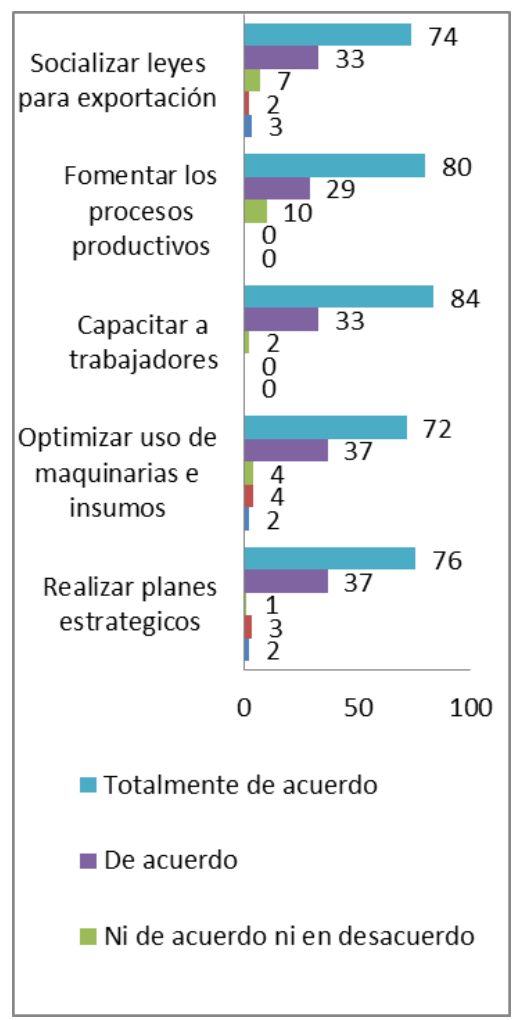


Fig. 3: Ilustración del nivel de insuficiencia en conocimientos administrativos de los empresarios

Se revalidan los resultados ilustrados en la figura 3, atendiendo al nivel de instrucción que tienen los empresarios en la conducción de las 119 pymes tomadas como muestras y que son objeto de investigación, las mismas que conciben las siguientes derivaciones:

i. Capacitación a los trabajadores (nivel operativo, táctico y gerencial) con 84 empresarios que manifiestan la necesidad de mejorar sus conocimientos.

ii. Fomentar los procesos productivos (insumos-proceso-producto) con 80 empresarios que declaran la necesidad de actualizarse en este trascendental tema.

iii. Realizar planes estratégicos (diagnostico-plan-acción-indicadores) con 76 empresarios que indican el insuficiente conocimiento en este tema.

iv. Socializar leyes para promover exportación (política gubernamental) con 74 que empresarios que manifiestan la necesidad de conocer los mecanismos que ofrece el gobierno para realizar comercialización externa

v. Optimizar uso de maquinarias e insumos (mejorar los mecanismos de control) con 72 empresarios que expresan la necesidad de precautelar los recursos de la empresa.

\section{Conclusiones}

En el presente reporte científico se reflejan los principales resultados obtenidos en la contextualización e implementación del Diagnóstico Organizacional, aplicando métodos empíricos que sustentan al Proyecto de Investigación "Estrategias Empresariales para lograr desarrollo productivo en los negocios fabriles de la provincia Santa Elena”. En el cuerpo de la memoria escrita del reporte, se describe la metodología utilizada que puede servir de referente a la comunidad científica, que investigan en las ramas del saber de las Ciencias Empresariales. Los autores del trabajo consideran que el aporte práctico de este artículo científico está dirigido a: 1) identificar las principales dificultades empresariales vertidas de los administradores de 119 negocios de un total de 653 empresas fabriles de la provincia Santa Elena 2) el nivel de instrucción de 119 empresarios de los Cantones de Santa Elena, La Libertad y Salinas, que inciden en la marcha del negocio 3) estos estadígrafos permitirán al equipo de investigadores a elaborar e implementar las estrategias empresariales que contribuyan a darle solución a la problemática científica, en función de lograr un verdadero desarrollo empresarial en los negocios fabriles de la provincia Santa Elena Ecuador.

\section{Bibliografía}

Bateman T, 2009. Administración, liderazgo y colaboración en un mundo competitivo (Octava edición) McGraw-Hill gerenciaestrategicaempresarial.wikispaces.com/file/detail/, Sitio web. Recuperado Diagnostico\%20empresarial.docx Consultado el 24 de septiembre del 2015

Hernández, S., y Rodríguez G., 2012 “Administración: Teoría, proceso, áreas funcionales y estrategias para la competitividad" Mc Graw Hill

Koontz \& O’Donnell, 2009 “Administración moderna (Octava edición) McGraw-Hill 
Meza, A. Carballeda, P., 2015 El diagnostico organizacional: elementos, métodos y técnicas. Recuperado de http://www.infosol.com.mx/espacio/Articulos/Desde_la_Investigacion/ElDiagnostico-Organizacional-elementos.html\#.Vg2UjiF_Oko Consultado el 22 de Septiembre del 2015

Oakland J., 2009. Administración por calidad total. (Primera edición) Editorial Continental

Ponce H., 2006. La matriz FODA: Una alternativa para realizar Diagnósticos y Determinar Estrategias de Intervención en las Organizaciones Productivas y Sociales. ESCA Santo Tomás. Contribuciones a la Economía. http://www.eumed.net/ce/

Robbins S, y Coulter M., 2009“Administración” Pearson Educación. ISBN 978-607-442-388-4 Pág.166 (2010)

Romagnoli S, 2012 Columna económica. Herramientas de Gestión: Diagnóstico Empresarial. Recuperado https://www.clubensayos.com/Temas-Variados/PlanificacionEducaticativa/187240.html Consultado el 22 de septiembre del 2015

Varela R., 2009 Innovación Empresarial. (Primera edición) Prentice Hall 